Historic, Archive Document

Do not assume content reflects current scientific knowledge, policies, or practices. 

Reserve

aQL676

.56

. K2B 57

1992

\section{BIRDS OF THE CIMARRON NATIONAL GRASSLAND}

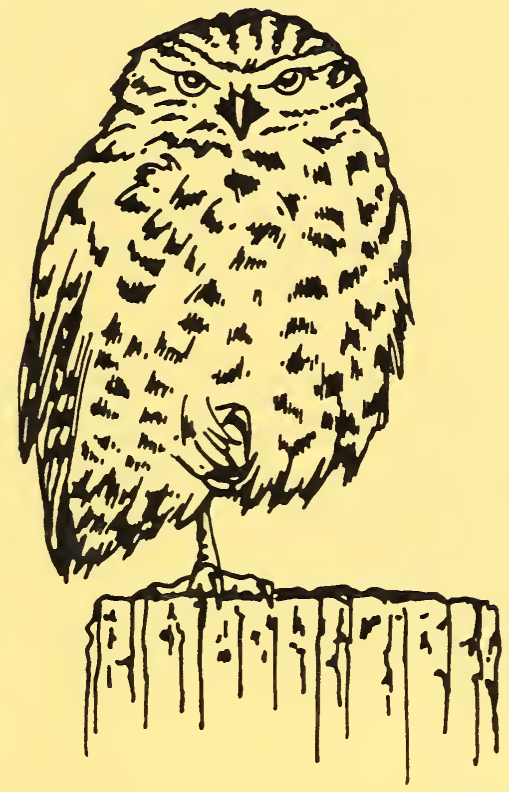

10 FEB 1993

PIKE AND SAN ISABEL

NATIONAL FORESTS

CIMARRON \& COMANCHE NATIONAL GRASSLANDS

U.S.D.A. FOREST SERVICE

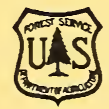


Birdw

Certai

birdin

all lev.

from s

travel,

The fo Kanse its bo 108,1: habita

ularity. ct that

$s$ and taken

$s$ you

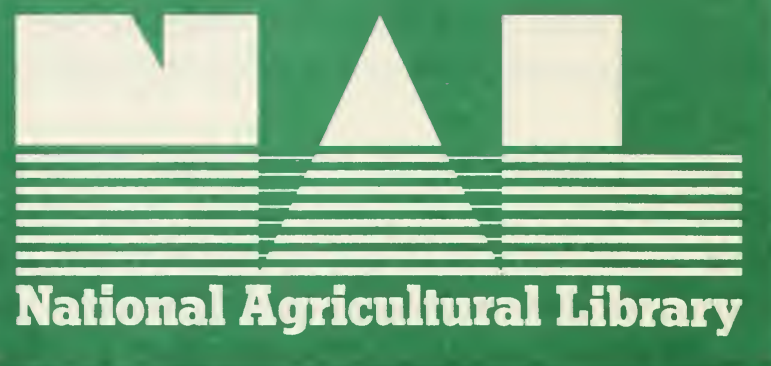

sunty, within and's ir bird ə bird ar this check checklist as your invitation to discover the pleasures and rewards of getting to know the birds of our National Grassland.

Bird records have been compiled by: Kansas Ornithological Society Record Committee

Kansas Department of Wildlife and Parks

Cimarron National Grassland

Ted Cable, Charles Ely, Earl McHugh Sebastian Patti, Mike Rader, Marvin Schwilling Scott \& Diane Seltman, Lawrence \& Ruth Smith

For additional information, please contact:

Cimarron National Grassland

242 E. Highway 56, P.O. Box J

Elkhart, KS 67950 316/697-4621

Additional sightings should be sent to:

Kansas Ornithological Society

Museum of Natural History

University of Kansas

Lawrence, KS 66045 
The following symbols and definitions are used:

BREEDING STATUS

* - Definite nesting records

\# - Probable nester

HABITAT USED

$\mathrm{H}$ - Hardlands (shortgrass)

$S$ - Sandylands (midgrass)

R - Riparian

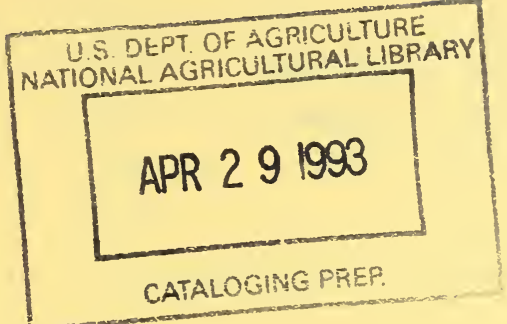

SEASONAL ABUNDANCE

$S$ - Spring (March through May)

$S$ - Summer (June through August)

F - Fall (September through November)

W - Winter (December through February)

\section{RELATIVE ABUNDANCE}

a - abundant, easily observed

c - common, certain to be seen in suitable habitat

$\mathrm{u}$ - uncommon, usually seen in suitable habitat

$r$ - rare, has been recorded at intervals of 2-5 years

$x$ - accidental, has been recorded only once or twice

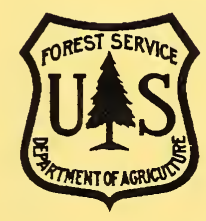


Common Loon

GREBES

Pied-billed Grebe

Horned Grebe

Eared Grebe

PELICANS

American White Pelican

CORMORANTS

Double-crested Cormorant

BITTERNS \& HERONS

American Bittern

Great Blue Heron

Great Egret

Snowy Egret

Cattle Egret

*Green-backed Heron

\#Black-crowned Night-Heron

Yellow-crowned Night-Heron

R

$x$

R

$R$

R

u

u

u

$r$

u

H

R

$R$

$R$

R

$\mathrm{R}, \mathrm{H}$

R

R

$R$

R

IBISES

White-faced Ibis

$\mathrm{R}, \mathrm{H}$

SWANS, GEESE \& DUCKS

Fulvous Whistling-Duck

Tundra Swan

_ Greater White-fronted Goose

- Snow Goose

Canada Goose

Wood Duck

Green-winged Teal

*Mallard

Northern Pintail

Blue-winged Teal

Cinnamon Teal

Northern Shoveler

Gadwall

$R$

$R$

$R$

$R$

$\mathrm{R}, \mathrm{H}$

$\mathrm{R}$

$R$

$R$

R

$R$

$R$

$R$

R 


\begin{tabular}{|c|c|c|c|c|c|}
\hline SPECIES & HABITAT & $\mathrm{s}$ & $\mathrm{s}$ & $\mathrm{F}$ & W \\
\hline American Wigeon & $R$ & $\mathrm{u}$ & & c & u \\
\hline Canvasback & $R$ & u & & u & u \\
\hline Redhead & $R$ & u & & u & u \\
\hline Ring-necked Duck & $R$ & u & & u & \\
\hline Greater Scaup & $R$ & $r$ & & & \\
\hline Lesser Scaup & $R$ & $\mathrm{u}$ & & $\mathrm{u}$ & \\
\hline Black Scoter & $R$ & & & $r$ & \\
\hline Surf Scoter & $\mathrm{R}$ & & & $r$ & \\
\hline White-winged Scoter & $R$ & & & $r$ & \\
\hline Common Goldeneye & $\mathrm{R}$ & & & & $r$ \\
\hline Bufflehead & $R$ & $u$ & & $r$ & $\mathrm{r}$ \\
\hline C Common Merganser & $R$ & & & & $r$ \\
\hline _ Red-breasted Merganser & $\mathrm{R}$ & & & & $\mathrm{x}$ \\
\hline _ Ruddy Duck & $R$ & $c$ & $\mathrm{u}$ & c & $r$ \\
\hline VULTURES & & & & & \\
\hline __ Turkey Vulture & $H, S, R$ & c & c & c & \\
\hline KITES, HAWKS, EAGLES \& ALLIES & & & & & \\
\hline Osprey & $\mathrm{R}$ & u & & u & \\
\hline${ }^{*}{ }^{\star}$ Mississippi Kite & $\mathrm{R}$ & c & c & u & \\
\hline Bald Eagle & $H, S, R$ & & & $\mathrm{u}$ & u \\
\hline Northern Harrier & $H, S, R$ & c & u & c & c \\
\hline \#Sharp-shinned Hawk & $H, R$ & u & & u & u \\
\hline Cooper's Hawk & $H, S, R$ & u & & u & u \\
\hline _ Northern Goshawk & & & & & $\mathrm{r}$ \\
\hline Red-shouldered Hawk & $\mathrm{R}$ & $x$ & & & $x$ \\
\hline Broad-winged Hawk & $H, S, R$ & $r$ & & $r$ & \\
\hline — & $H, S, R$ & c & c & c & \\
\hline - Red-tailed Hawk & $H, S, R$ & c & c & c & c \\
\hline —Ferruginous Hawk & $H, S$ & c & u & c & c \\
\hline Z Rough-legged Hawk & $H, S, R$ & u & & u & c \\
\hline __ Golden Eagle & $H, S, R$ & u & $r$ & u & u \\
\hline FALCONS & & & & & \\
\hline${ }^{\star}$ American Kestrel & $H, S, R$ & c & c & c & c \\
\hline - Merlin & $H, S, R$ & u & & u & u \\
\hline Peregrine Falcon & $H, S, R$ & $r$ & & $r$ & $r$ \\
\hline _Prairie Falcon & $H, S, R$ & u & $\mathrm{r}$ & u & c \\
\hline
\end{tabular}


PARTRIDGES, GROUSE, TURKEY, QUAIL

*Ring-necked Pheasant

*Lesser Prairie-Chicken

*Wild Turkey

*Northern Bobwhite

*Scaled Quail

RAILS \& COOTS

\section{Black Rail}

*Virginia Rail

*Sora

*American Coot

\section{CRANES}

Sandhill Crane

\section{PLOVERS}

Black-bellied Plover

Snowy Plover

Semipalmated Plover

*Killdeer

Mountain Plover

STILTS \& AVOCETS

Black-necked Stilt

American Avocet

SANDPIPERS, PHALAROPES \& ALLIES Greater Yellowlegs Lesser Yellowlegs Solitary Sandpiper Spotted Sandpiper \#Upland Sandpiper Whimbrel

*Long-billed Curlew Marbled Godwit Ruddy Turnstone Sanderling Semipalmated Sandpiper Western Sandpiper

$H, S, R$

$H, S, R$

$R$

R

$R$

$R$

$\mathrm{R}$

$\mathrm{R}$

$H, S, R$

$H, S, R$

$H, S, R$

$S, R$

$R$

R

R 
Least Sandpiper

White-rumped Sandpiper

Baird's Sandpiper

Pectoral Sandpiper

Stilt Sandpiper

Buff-breasted Sandpiper

Long-billed Dowitcher Common Snipe

Wilson's Phalarope

Red-necked Phalarope

Red Phalarope

GULLS \& TERNS

Franklin's Gull

Bonaparte's Gull

Ring-billed Gull

California Gull

Herring Gull

Sabine's Gull

Forster's Tern

Black Tern

PIGEONS \& DOVES

*Rock Dove

*Mourning Dove

CUCKOOS \& ROADRUNNERS

Black-billed Cuckoo

*Yellow-billed Cuckoo

Greater Roadrunner

BARN OWLS

*Barn Owl

TYPICAL OWLS

*Eastern Screech-Owl

\#Western Screech-Owl

*Great Horned Owl

Snowy Owl

*Burrowing Owl

*Long-eared Owl

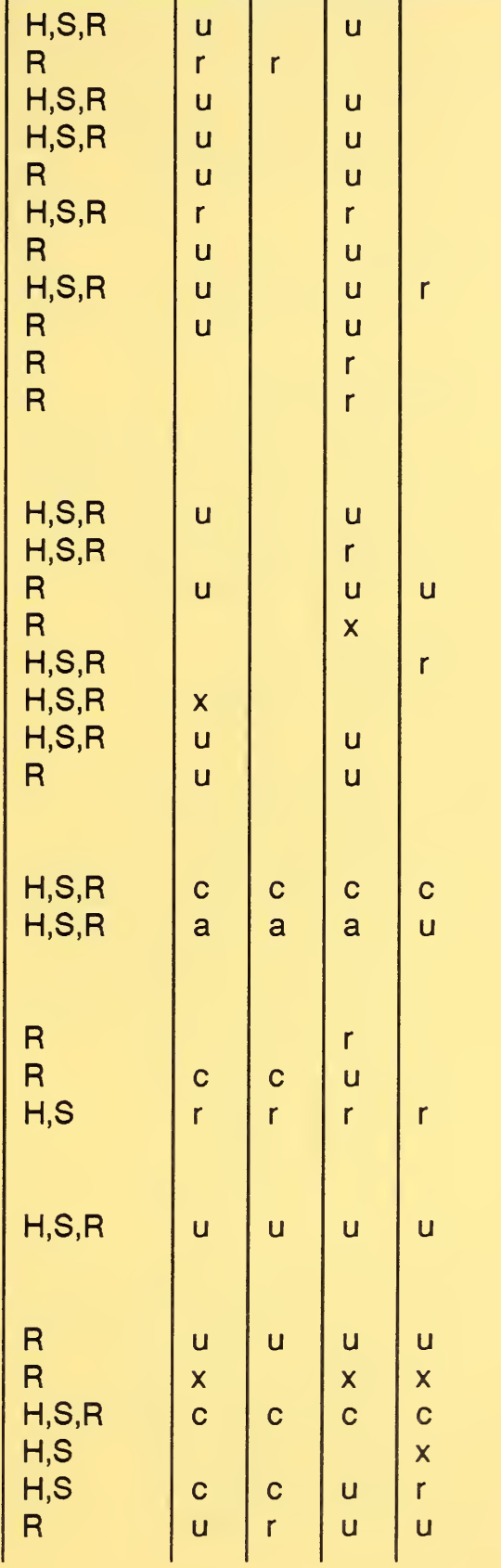




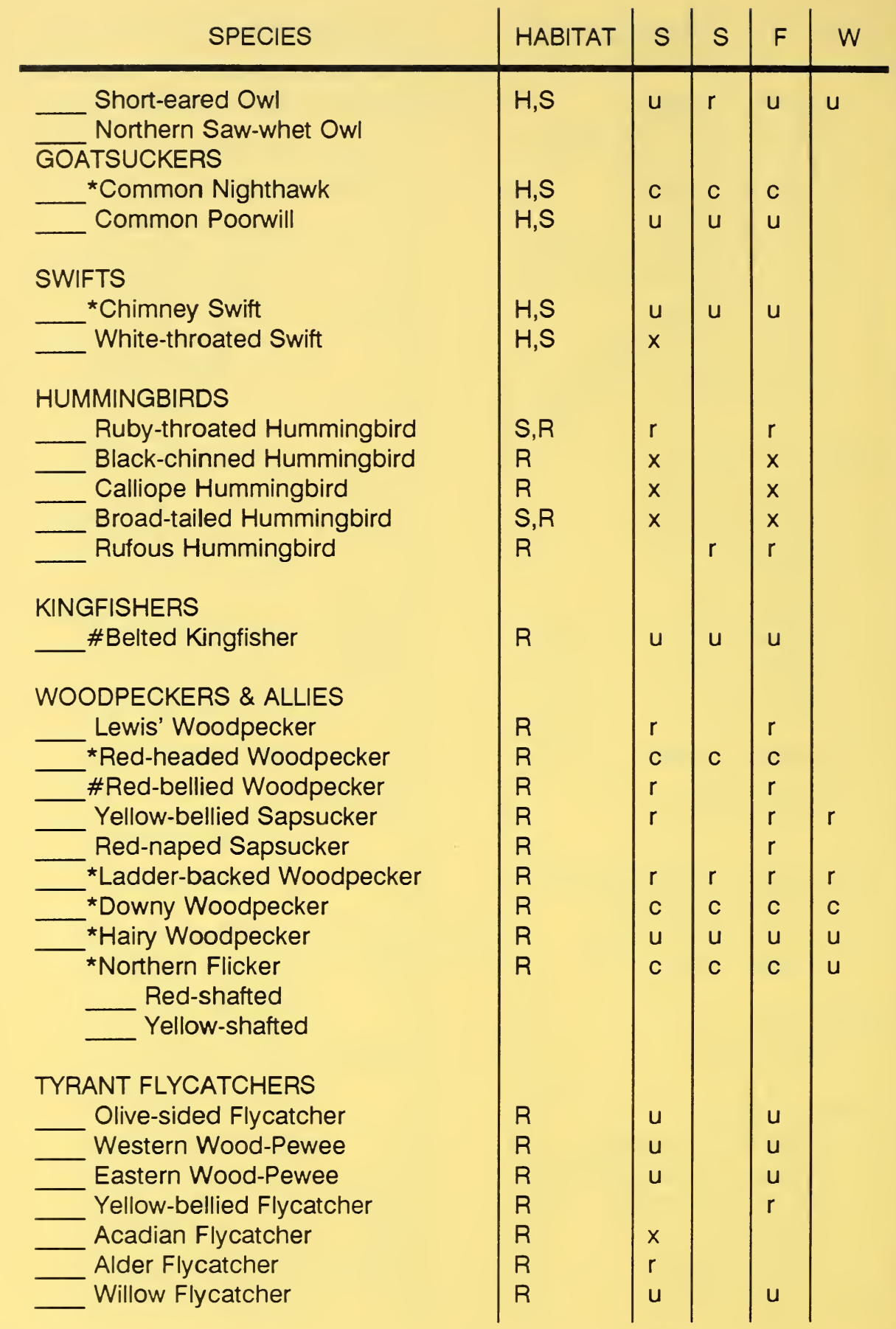


Least Flycatcher

Hammond's Flycatcher

Dusky Flycatcher

Gray Flycatcher

Black Phoebe

Cordilleran Flycatcher

*Eastern Phoebe

\#Say's Phoebe

Vermilion Flycatcher

*Ash-throated Flycatcher

*Great Crested Flycatcher

Cassin's Kingbird

*Western Kingbird

*Eastern Kingbird

*Scissor-tailed Flycatcher

\begin{tabular}{l|l|l|l|l}
$R$ & $u$ & & $u$ & \\
$R$ & & & $r$ & \\
$R$ & $r$ & & $r$ & \\
$R$ & $x$ & & $x$ & \\
$H$ & $x$ & & & \\
$R$ & $r$ & & $r$ & \\
$R$ & $u$ & $u$ & $u$ & \\
$H, S$ & $c$ & $u$ & $c$ & $r$ \\
$R$ & $x$ & & & \\
$R$ & $u$ & $r$ & $u$ & \\
$R$ & $u$ & $u$ & $u$ & \\
$H, S, R$ & $u$ & & $u$ & \\
$H, S, R$ & $a$ & $a$ & $c$ & \\
$H, S, R$ & $c$ & $c$ & $c$ & \\
$H, S, R$ & $u$ & $u$ & $u$ & \\
& & & &
\end{tabular}

*Horned Lark

$H, S, R$

a

a

a

SWALLOWS

Purple Martin

Tree Swallow

Violet-green Swallow

*Northern Rough-winged Swallow

Bank Swallow

*Cliff Swallow

^Barn Swallow

JAYS, MAGPIES \& CROWS

Steller's Jay

^Blue Jay

Scrub Jay

Pinyon Jay

*Black-billed Magpie

*American Crow

${ }^{\star}$ Chihuahuan Raven

TITMICE

^Black-capped Chickadee Mountain Chickadee

\begin{tabular}{l|l|l|l|l}
$H, S, R$ & $u$ & $u$ & & \\
$R$ & $u$ & & $u$ & \\
$H, R$ & & & $r$ & \\
$H, S, R$ & $c$ & $c$ & $c$ & \\
$H, S, R$ & $u$ & & $u$ & \\
$H, S, R$ & $u$ & $u$ & $u$ & \\
$H, S, R$ & $a$ & $a$ & $a$ & \\
& & & & \\
$R$ & $r$ & & $r$ & $r$ \\
$R$ & $c$ & $c$ & $c$ & $u$ \\
$R$ & $r$ & $r$ & $r$ & $r$ \\
$R$ & $r$ & & $r$ & \\
$H, S, R$ & $a$ & $a$ & $a$ & $a$ \\
$H, S, R$ & $c$ & $c$ & $c$ & $c$ \\
$H, S, R$ & $u$ & $r$ & $u$ & $r$ \\
& & & & \\
$R$ & $r$ & $r$ & $r$ & $r$ \\
$R$ & $r$ & $r$ & & $r$
\end{tabular}




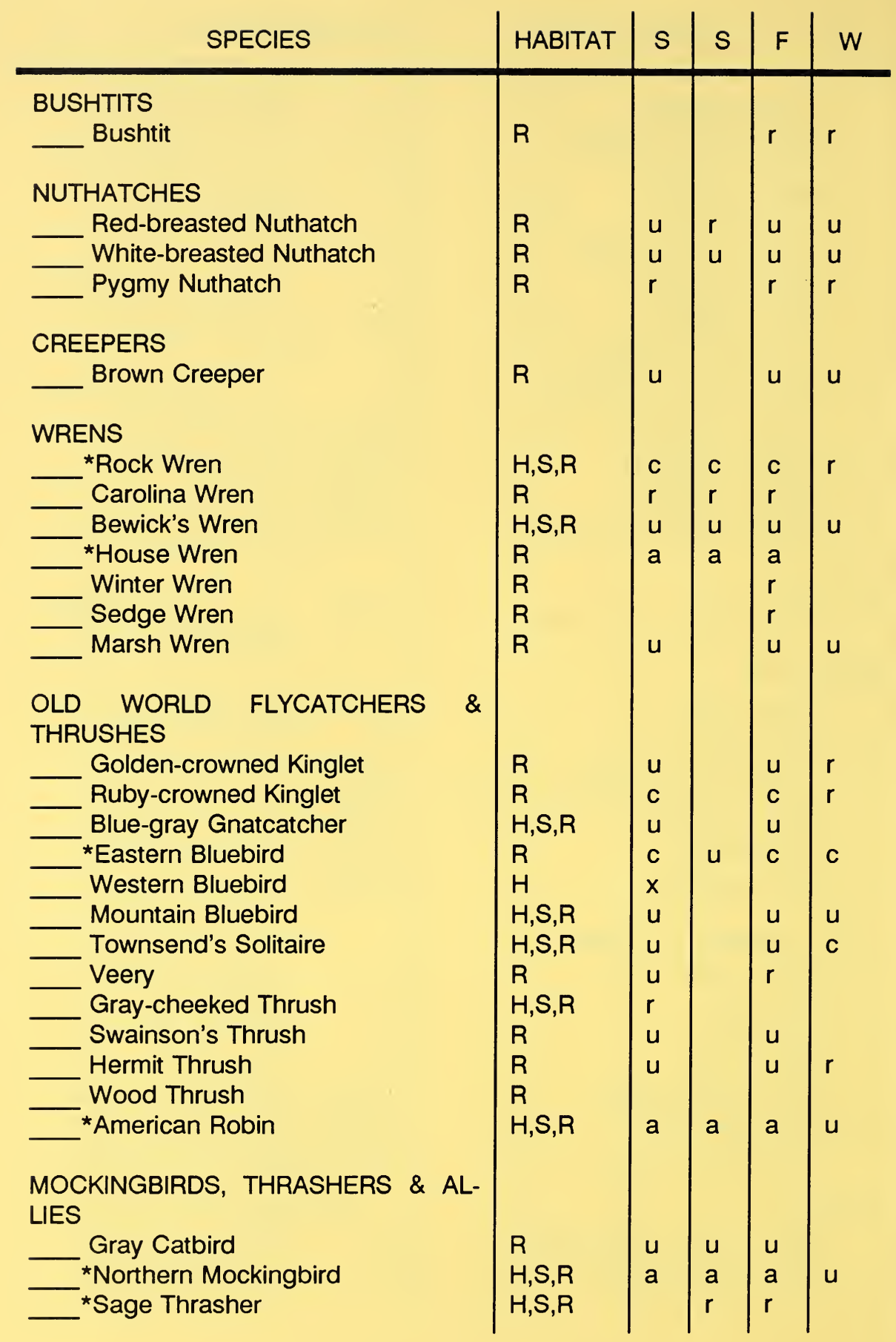


*Brown Thrasher

${ }^{\star}$ Curve-billed Thrasher

PIPITS

American Pipit

WAXWINGS

Bohemian Waxwing

Cedar Waxwing

SILKY-FLYCATCHERS

Phainopepla

\section{SHRIKES}

Northern Shrike

*Loggerhead Shrike

\section{STARLINGS}

*European Starling

\section{VIREOS}

White-eyed Vireo

\#Bell's Vireo

Solitary Vireo

Yellow-throated Vireo

\#Warbling Vireo

Philadelphia Vireo

\#Red-eyed Vireo

WOOD-WARBLERS, TANAGERS, CARDINALS, GROSBEAKS, EMBERIZINES, BLACKBIRDS \& ALLIES

Blue-winged Warbler

Golden-winged Warbler

Tennessee Warbler

Orange-crowned Warbler

Nashville Warbler

Virginia's Warbler

Northern Parula

\#Yellow Warbler

Chestnut-sided Warbler

\begin{tabular}{|c|c|c|c|}
\hline $\begin{array}{l}H, S, R \\
H, S\end{array}$ & $\begin{array}{l}c \\
r\end{array}$ & $\begin{array}{l}c \\
r\end{array}$ & $\begin{array}{l}c \\
r\end{array}$ \\
\hline$H, S, R$ & $\mathrm{u}$ & & $\mathrm{u}$ \\
\hline$R$ & & & \\
\hline$R$ & $u$ & & $u$ \\
\hline$H$ & & & $x$ \\
\hline$H, S, R$ & & & $r$ \\
\hline$H, S, R$ & c & $c$ & $\mathrm{c}$ \\
\hline$H, S, R$ & $a$ & a & a \\
\hline$R$ & $r$ & & \\
\hline$R$ & $u$ & $u$ & $u$ \\
\hline$R$ & $\mathrm{u}$ & & $\mathrm{u}$ \\
\hline$R$ & $x$ & & $x$ \\
\hline$R$ & c & c & C \\
\hline$R$ & $r$ & & \\
\hline$R$ & $u$ & $\mathrm{u}$ & $\mathrm{u}$ \\
\hline$R$ & $r$ & & \\
\hline$R$ & $r$ & & \\
\hline$H, S, R$ & $u$ & & $u$ \\
\hline$H, S, R$ & c & & c \\
\hline$H, S, R$ & $\mathrm{u}$ & & $\mathrm{u}$ \\
\hline$H, R$ & $x$ & $x$ & $x$ \\
\hline$H, S, R$ & $r$ & & \\
\hline$H, S, R$ & c & $\mathrm{u}$ & c \\
\hline$H, S, R$ & $x$ & & $r$ \\
\hline
\end{tabular}


Magnolia Warbler

Cape May Warbler

Black-throated Blue Warbler

Yellow-rumped Warbler Myrtle

\section{Audubon's}

Black-throated Gray Warbler Townsend's Warbler Black-throated Green Warbler Blackburnian Warbler Grace's

Pine Warbler Prairie Warbler

Palm Warbler

Bay-breasted Warbler Blackpoll Warbler Cerulean Warbler Black-and-white Warbler American Redstart Prothonotary Warbler Worm-eating Warbler Ovenbird Northern Waterthrush Louisiana Waterthrush Kentucky Warbler MacGillivray's Warbler \#Common Yellowthroat Hooded Warbler Wilson's Warbler \#Yellow-breasted Chat Summer Tanager Western Tanager Northern Cardinal Pyrrhuloxia Rose-breasted Grosbeak ^Black-headed Grosbeak *Blue Grosbeak *Lazuli Bunting *Indigo Bunting *Dickcissel Green-tailed Towhee

\begin{tabular}{l|l|l|l|l}
$H, S, R$ & $x$ & & & \\
$R$ & $r$ & & & \\
$H, S, R$ & $r$ & & $r$ & \\
$H, S, R$ & $a$ & & $a$ & $a$
\end{tabular}

$H, S, R$ $H, S, R$ $\mathrm{H}, \mathrm{S}, \mathrm{R}$ $S, R$ $R$ $\mathrm{H}, \mathrm{S}, \mathrm{R}$ $R$ $H, S, R$ $S, R$ $H, S, R$

$\mathrm{R}$

R

$H, S, R$ $H, S, R$

$R$

$H, S, R$

$R$

R

$H, S, R$ H,S, R $\mathrm{H}, \mathrm{S}, \mathrm{R}$

$R$

R

R

$H, S, R$

$H, S, R$

$S, R$

$R$

$H, S, R$

$H, S, R$

$R$

$H, R$

R

H,S

$H, R$ 
Rufous-sided Towhee Canyon Towhee *Cassin's Sparrow Rufous-crowned Sparrow American Tree Sparrow Chipping Sparrow *Clay-colored Sparrow *Brewer's Sparrow

*Field Sparrow Black-chinned Sparrow Vesper Sparrow *Lark Sparrow Black-throated Sparrow Sage Sparrow *Lark Bunting Savannah Sparrow Baird's Sparrow *Grasshopper Sparrow LeConte's Sparrow Fox Sparrow Song Sparrow Lincoln's Sparrow Swamp Sparrow White-throated Sparrow Golden-crowned Sparrow White-crowned Sparrow Harris' Sparrow Dark-eyed Juncos White-winged Slate-colored Oregon Gray-headed McCown's Longspur Lapland Longspur Smith's Longspur Chestnut-collared Longspur Bobolink

*Red-winged Blackbird Eastern Meadowlark *Western Meadowlark Yellow-headed Blackbird

$H, R$

$\mathrm{H}$

$\mathrm{H}, \mathrm{S}$

$\mathrm{H}$

$H, S, R$

$H, S$

$H, S, R$

$H, S$

$\mathrm{H}, \mathrm{S}$

$S$

$H, S$

$H, R, S$

$H, S$

$H, S$

$H, S$

$H, S, R$

$H, S$

$H, S$

$H, S, R$

$R$

$H, S, R$

$R$

$\mathrm{H}, \mathrm{S}, \mathrm{R}$

$S, R$

$\mathrm{H}$

$H, S, R$

$R$

$H, S, R$

\begin{tabular}{ll|l|l|l|l} 
& & & & \\
$H$ & & & & \\
$H, S, R$ & $c$ & & $u$ & $a$ \\
$H$ & & & $x$ & \\
$S$ & $c$ & & $u$ & $c$ \\
$S$ & $r$ & & & \\
$S, R$ & $a$ & $a$ & $a$ & $c$ \\
$H$ & $x$ & $u$ & $u$ & \\
$H, S, R$ & $a$ & $a$ & $a$ & $a$ \\
$R$ & $c$ & $r$ & $c$ &
\end{tabular}




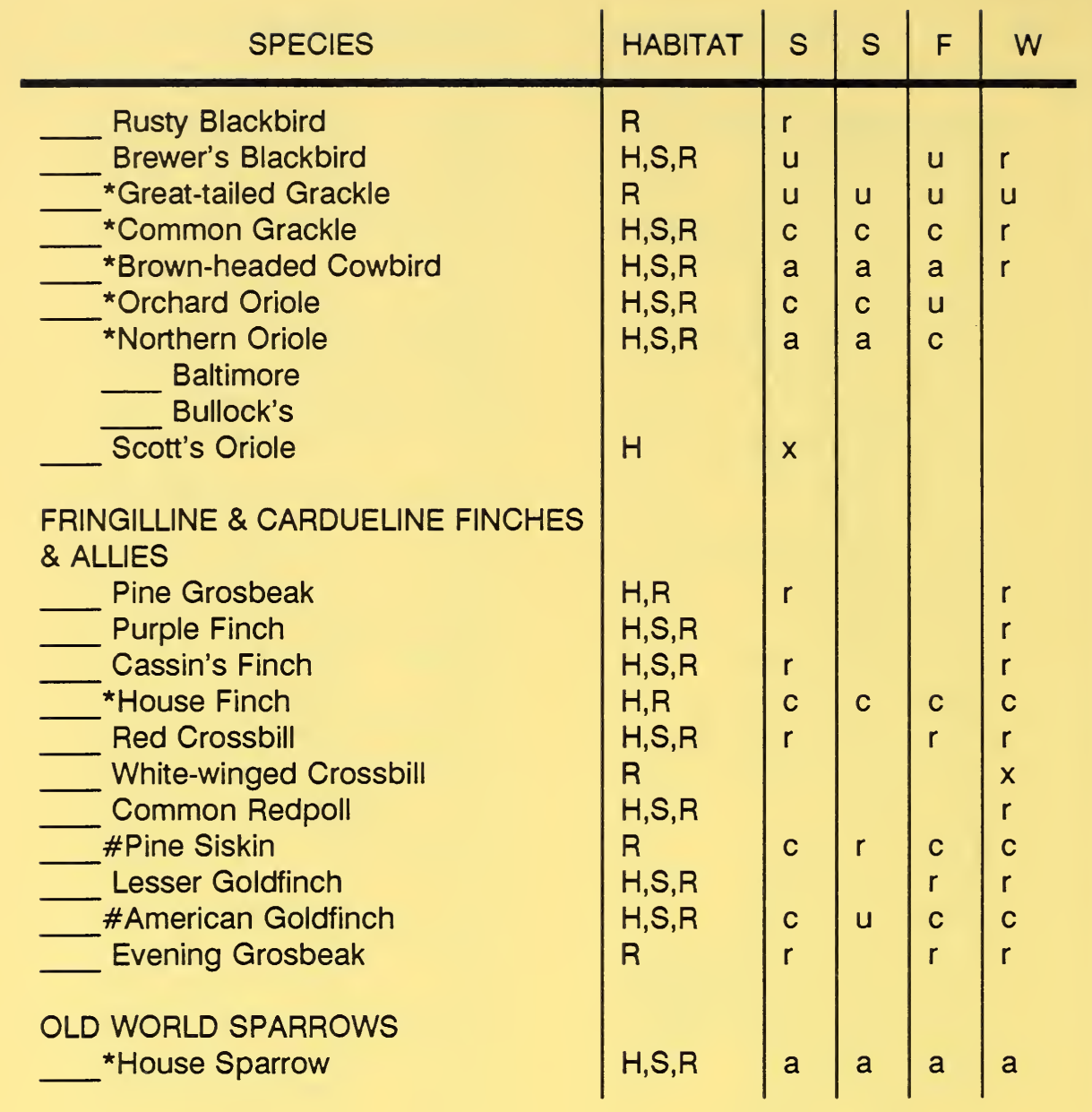


